Inaccurate

\section{assessment of \\ canine body condition \\ score, bodyweight \\ and pet food labels: \\ a cause for \\ concern}

\section{Amanda Root, Christina Butowski, G. Naughton, Philippa Yam}

University of Glasgow, Glasgow, Scotland, UK

\section{OBJECTIVES}

To investigate owners ability to assign the correct bodyweight (BW) and body condition score (BCS) to their dog, and owners ability to interpret wet and dry pet food labels by estimating how much to feed daily.

\section{METHODS}

One hundred and seventy-four questionnaires were completed by dog owners during 2014 and 2015. The interviewer's assessment of BCS and BW of each dog was taken as the gold standard. Owner estimated BW was considered correct if within $10 \%$ of actual BW. Correct interpretation of daily wet/dry food allowance was assessed by determining the number of cans (wet food) and grams (dry food) required $+20 \%$ based on the dog's actual BW and feeding guidelines on the label compared to the owner's estimate.

\section{RESULTS}

Thirty-eight percent of dogs were overweight/obese. Owner under-estimation of BCS (38\%) and BW $(33 \%)$ was the most common misperception. Eleven percent of owners over-estimated BCS and 19\% over-estimated BW. Only $23 \%$ and $43 \%$ of owners for wet and dry food, respectively were able to correctly estimate amount to feed on a daily basis. The current findings of misperception substantiate those previously reported in veterinary literature.

\section{STATEMENT}

Many owners are not aware of their pet's BCS and BW and cannot accurately interpret pet food labels. This highlights the continuing need for owner education to improve these skills if dogs are to be fed correctly.

\section{Sialolipoma of the parotid salivary gland in two dogs}

\section{Veronica Rodiño Tilve, Marlene Finck, Joshua Leach, Michael Macfarlane}

School of Veterinary Medicine, Glasgow, UK

\section{OBJECTIVES}

To report the presentation, diagnostic approach, surgical treatment and outcome of two dogs with an unreported condition of the parotid salivary gland.

\section{METHODS}

Case-series describing two cases of unilateral sialolipoma of the parotid salivary gland in the dog. The diagnostic approach, treatment, outcome and prognosis are outlined with consistent comparison with human medicine literature.

\section{RESULTS}

Both dogs presented for investigation of a soft, relatively moveable, non-painful subcutaneous mass at the level of the right parotid salivary gland. Fine needle aspirates of the masses yielded adipose cells and normal salivary epithelium. Contrast-enhanced computed tomography of both masses showed a mixed fat attenuating mass in the region of the right parotid salivary gland, without a normal ipsilateral parotid salivary gland. Biopsy sample collected from one of the masses was suggestive of sialolipoma. Diagnosis is made based on the presence of a surrounding fibrous capsule. Parotid gland sialadenectomy was performed and definitive diagnosis achieved by histopathology. No recurrence was identified seven and sixteen months post-operatively.

\section{STATEMENT}

As there are no previous reports of this tumour in this location, dogs with masses in the region of the parotid gland, which are not consistent with a sialocele or an abscess, are often considered to have a poor prognosis. Extrapolating from human literature, the prognosis for sialolipoma would be expected to be favourable after surgical excision. It is hoped that this case report will result in increased awareness of this uncommon neoplasm. 